\title{
Correspondence on "G-CSF as a suitable alternative to GM-CSF to boost dinutuximab-mediated neutrophil cytotoxicity in neuroblastoma treatment" by Martinez Sanz et al
}

To cite: Mora J, Chantada GL. Correspondence on "G-CSF as a suitable alternative to GM-CSF to boost dinutuximabmediated neutrophil cytotoxicity in neuroblastoma treatment" by Martinez Sanz et al. Journal for ImmunoTherapy of Cancer 2021;9:e003751. doi:10.1136/ jitc-2021-003751

Accepted 07 September 2021

\section{Linked}

http://dx.doi.org/10.1136/ jitc-2021-003983

http://dx.doi.org/10.1136/ jitc-2020-002259

Check for updates

(C) Author(s) (or their employer(s)) 2021. Re-use permitted under CC BY-NC. No commercial re-use. See rights and permissions. Published by BMJ.

${ }^{1}$ Pediatric Cancer Center Barcelona, Hospital Sant Joan de Déu, Barcelona, Spain

${ }^{2}$ Pereira Rossell Hospital Center, Montevideo, Uruguay

${ }^{3}$ Hospital Universitario Austral, Pilar, Argentina

Correspondence to

Dr Jaume Mora;

jaume.mora@sjd.es
Dear Editor,

We read with great interest the work by Martinez Sanz et $a l^{1}$ 'G-CSF as a suitable alternative to GM-CSF to boost dinutuximabmediated neutrophil cytotoxicity in neuroblastoma treatment,' published in the Journal for ImmunoTherapy of Cancer on May 28, 2021. The authors note access to recombinant human granulocyte-macrophage colony-stimulating factor (rhu GM-CSF; sargramostim (yeast-derived)) is limited outside of North America, potentially leading to suboptimal treatment of patients with neuroblastoma and therefore necessitating an alternative agent to stimulate dinutuximab immunotherapy-responsiveness in the treatment of neuroblastoma. Using preclinical models, the study compared the efficacy of neutrophils stimulated with either GM-CSF or granulocyte colony-stimulating factor (G-CSF) to kill dinutuximab-opsonized GD2positive neuroblastoma cell lines and primary patient tumor material. G-CSF enhancement of neutrophil killing capacity of neuroblastoma cells was reported to be as potent as GM-CSF. The authors concluded that their in vitro study, along with other preclinical and small clinical studies, justifies the study of G-CSF as a potentially suitable alternative for sargramostim in patients with neuroblastoma.

Currently, there are limited clinical data using G-CSF as part of the dinutuximab regimen. A recent phase I-IIa Japanese clinical study by Hara and coworkers ${ }^{2}$ involving 25 patients reported tolerability with G-CSF or M-CSF given in conjunction with dinutuximab in patients with high-risk neuroblastoma. The authors of this study, however, stated that their sample size was too small to confirm the safety of using G-CSF or M-CSF as part of the dinutuximab regimen. Further, the follow-up period of this trial was insufficient to evaluate a survival benefit.

Previous work has shown that G-CSF may not be an appropriate agent for patients with high-risk neuroblastoma, as neuroblastoma cell lines express mRNA for the G-CSF receptor and are stimulated in response to exogenous G-CSF, leading to a more aggressive biologic behavior and increased invasiveness. Martinez Sanz et al reported in vitro G-CSF did not show increased neuroblastoma cell growth or a change in the susceptibility of neuroblastoma to neutrophil-mediated cytotoxicity as compared with the control, and no evidence of effect on the neuroblastoma phenotype.

GM-CSF stimulates production of granulocytes, monocytes/macrophages, and promotes maturation of monocytes to antigenpresenting cells. Granulocytes are important contributors to antitumor responses when using combination therapy of anti-GD2 antibodies and sargramostim. ${ }^{3}$ Increased circulating myeloid cells alter the tumor microenvironment and lead to improved T-cell response. ${ }^{4}$ Additionally, GM-CSF polarizes macrophages to the M1 phenotype which can lead to increased tumor necrosis factor. The safety and efficacy of GM-CSF has been well established in patients with high-risk neuroblastoma and is indicated in combination with dinutuximab and naxitamab-gqgk (see Unituxan and Danyelza prescribing information).

Martinez Sanz et al highlight the critical need for access to sargramostim for all patients with high-risk neuroblastoma. The benefits of combining anti-GD2 antibody and sargramostim therapy are well known, 
but since worldwide access is limited and sometimes cost prohibitive, the focus of research outside of North America has shifted to identifying alternatives. Patients with high-risk neuroblastoma deserve access to the best treatment options available and adapted to the local resources and should not have to settle for alternative therapeutic options, regardless of where they reside. In fact, research studies addressing setting-relevant questions need to be performed also in low-income and middle-income countries. ${ }^{5}$

Understandably, there is urgency for identifying alternatives to sargramostim for clinical use outside of North America, but we urge caution in using G-CSF as an alternative without further study, as these agents are not interchangeable. Healthcare providers need to continue to advocate for our patients and focus on the goal of providing affordable access to the most effective therapies for all patients with high-risk neuroblastoma.

Sincerely,

Dr Jaume Mora and Dr Guillermo Chantada

Contributors All authors reviewed, revised, and approved this letter.

Funding Partner Therapeutics, Inc. provided medical writing support, approved submission, and funded article processing charges for this letter.

Competing interests Outside the current work, JM has received consulting fees and payment or honoraria for educational activities from Y-mAbs Therapeutics, Inc., and GC has received honoraria for educational activities from Y-mAbs Therapeutics, Inc. and consulting fees from Adium Pharma.

Patient consent for publication Not applicable.

Provenance and peer review Commissioned; internally peer reviewed.

Open access This is an open access article distributed in accordance with the Creative Commons Attribution Non Commercial (CC BY-NC 4.0) license, which permits others to distribute, remix, adapt, build upon this work non-commercially, and license their derivative works on different terms, provided the original work is properly cited, appropriate credit is given, any changes made indicated, and the use is non-commercial. See http://creativecommons.org/licenses/by-nc/4.0/.

\section{REFERENCES}

1 Martinez Sanz P, van Rees DJ, van Zogchel LMJ, et al. G-CSF as a suitable alternative to GM-CSF to boost dinutuximab-mediated neutrophil cytotoxicity in neuroblastoma treatment. J Immunother Cancer 2021;9:e002259.

2 Hara J, Nitani C, Kawamoto $\mathrm{H}$, et al. A phase I/Ila study of antidisialoganglioside antibody dinutuximab in Japanese patients with neuroblastoma. J Pediatr Hematol Oncol 2021;43:e358-64.

3 Cheung IY, Hsu K, Cheung N-KV. Activation of peripheral-blood granulocytes is strongly correlated with patient outcome after immunotherapy with anti-GD2 monoclonal antibody and granulocytemacrophage colony-stimulating factor. J Clin Oncol 2012;30:426-32.

4 Mody R, Yu AL, Naranjo A, et al. Irinotecan, temozolomide, and Dinutuximab with GM-CSF in children with refractory or relapsed neuroblastoma: a report from the children's Oncology Group. J Clin Oncol 2020;38:2160-9.

5 Van Heerden J, Kruger M. The need for resource-linked translational oncology in the management of neuroblastoma and other childhood malignancies in low- and middle-income countries. Pediatr Blood Cancer 2021;68:e29133. 